\begin{tabular}{|c|c|}
\hline Title & $\begin{array}{l}\text { Neuronal Correlates of Multiple Top-Down Signals s during Covert Tracking of Moving Objects in Macaque Prefrontal } \\
\text { Cortex }\end{array}$ \\
\hline Author(s) & Matsushima, A yano; Tanaka, Masaki \\
\hline Citation & $\begin{array}{l}\text { Journal of Cognitive Neuroscience, 24(10), } 20432056 \\
\text { https://doi.org/10.1162 Jocn_a_00265 }\end{array}$ \\
\hline Issue Date & $2012-10$ \\
\hline Doc URL & http:/hdl. handle.net/2115/51034 \\
\hline Rights & (c) 2012 Massachusetts Institute of Technology \\
\hline Type & article \\
\hline File Information & JCN24-10_2043-2056.pdf \\
\hline
\end{tabular}

Instructions for use 


\title{
Neuronal Correlates of Multiple Top-Down Signals during Covert Tracking of Moving Objects in Macaque Prefrontal Cortex
}

\author{
Ayano Matsushima and Masaki Tanaka
}

\begin{abstract}
Resistance to distraction is a key component of executive functions and is strongly linked to the prefrontal cortex. Recent evidence suggests that neural mechanisms exist for selective suppression of task-irrelevant information. However, neuronal signals related to selective suppression have not yet been identified, whereas nonselective surround suppression, which results from attentional enhancement for relevant stimuli, has been well documented. This study examined single neuron activities in the lateral PFC when monkeys covertly tracked one of randomly moving objects. Although many neurons responded to the target, we also found a group of neurons that exhibited a selective response
\end{abstract}

\section{INTRODUCTION}

Selective attention is a neural mechanism to highlight the relevant information for perception and behavior (Corbetta \& Shulman, 2002; Luck, Woodman, \& Vogel, 2000; Posner \& Petersen, 1990). Previous studies have shown that visual responses to task-relevant objects are enhanced (Chen et al., 2008; Polk, Drake, Jonides, Smith, \& Smith, 2008; McAdams \& Maunsell, 1999; Hillyard, Vogel, \& Luck, 1998; Luck, Chelazzi, Hillyard, \& Desimone, 1997; Schall \& Hanes, 1993), whereas that to the other objects are suppressed (Sundberg, Mitchell, \& Reynolds, 2009; Chen et al., 2008; Polk et al., 2008; Motter, 1994; Schall \& Hanes, 1993; Moran \& Desimone, 1985). According to the present theories of attention (Reynolds \& Heeger, 2009; Desimone \& Duncan, 1995), the nonselective inhibition of irrelevant information is a byproduct of the enhancement of relevant information by virtue of local competition between conflicting representations.

Although most studies of selective attention have focused on the facilitation of relevant information, there is growing evidence that irrelevant information is not only passively filtered out but is also actively suppressed. Recent psychophysical studies have shown that detection of a probe stimulus presented at distractor locations is degraded compared with empty space, when subjects perform visual search tasks (Thomas \& Lleras, 2009; Muller

Hokkaido University School of Medicine, Sapporo, Japan to the distractor that was visually identical to the target. Because most neurons were insensitive to an additional distractor that explicitly differed in color from the target, the brain seemed to monitor the distractor only when necessary to maintain internal object segregation. Our results suggest that the lateral PFC might provide at least two top-down signals during covert object tracking: one for enhancement of visual processing for the target and the other for selective suppression of visual processing for the distractor. These signals might work together to discriminate objects, thereby regulating both the sensitivity and specificity of target choice during covert object tracking.

\& von Muhlenen, 2000; Watson \& Humphreys, 2000; Klein, 1988) or when subjects mentally track several moving objects in a multiple object tracking task (Flombaum, Scholl, \& Pylyshyn, 2008; Pylyshyn, Haladjian, King, \& Reilly, 2008; Pylyshyn, 2006; Ogawa, Takeda, \& Yagi, 2002; Pylyshyn \& Storm, 1988). These results indicate that visual processing at distractor locations is selectively inhibited irrespective of target locations, suggesting that the brain monitors the distractors separately from the targets (Flombaum et al., 2008). Importantly, the difficulties in probe detection at distractor locations have not been observed when subjects simply viewed the stimuli (Thomas \& Lleras, 2009; Pylyshyn et al., 2008; Pylyshyn, 2006) or when the distractors did not interfere with task performance (Pylyshyn et al., 2008; Cepeda, Cave, Bichot, \& Kim, 1998), suggesting that the suppression of distractor processing is not an automatic process but rather under deliberate control. All of these previous findings indicate the existence of top-down signals for selective suppression of the distractor that are distinct from the signals highlighting the target.

To explore top-down signals for monitoring of individual objects, we examined single neuron activities in the lateral PFC when monkeys covertly tracked one of the identical, moving objects without eye movements. Consistent with the previous studies conducted in V4 of monkeys (Sundberg et al., 2009; Mitchell, Sundberg, \& Reynolds, 2007), we found that the majority of neurons in the lateral PFC modulated their activities depending mostly on the 
target location. In addition, we also found a novel type of neurons that exhibited greater firing modulation for the distractor than the target when and only when the distractor was identical to the target, suggesting that those signals were necessary to internally discriminate the objects. We propose that the signals monitoring the target may enhance visual processing for the target (Moore \& Fallah, 2004; Moore \& Armstrong, 2003), whereas those monitoring the distractor may selectively suppress visual processing for the distractor, which has been demonstrated repeatedly by psychological experiments (Thomas \& Lleras, 2009; Pylyshyn et al., 2008; Muller \& von Muhlenen, 2000; Klein, 1988).

\section{METHODS}

Experiments were conducted on two Japanese macaques (Macaca fuscata, 6-7 kg female monkeys F and L). The animals were prepared for chronic recording experiments using sterile surgical procedures with isoflurane anesthesia. All animal protocols were approved in advance by the Animal Care and Use Committee of Hokkaido University and were in accord with the Guide for the Care and Use of Laboratory Animals.

\section{Visual Stimuli and Behavioral Paradigms}

Experiments were controlled by a Windows-based real-time data acquisition system (TEMPO; Reflective Computing). Visual stimuli were presented on a 24-in. cathode ray tube monitor (refresh rate: $60 \mathrm{~Hz}$ ) positioned $38 \mathrm{~cm}$ from the eyes and subtending $64 \times 44^{\circ}$ of visual angle. All visual stimuli were presented within a $40^{\circ}$ square contour that was presented on the center of the screen and was visible throughout the experiments. Experiments were carried out in a darkened booth. Horizontal and vertical eye position signals were obtained using the search coil technique (MEL-25; Enzanshi Kogyo) and were calibrated before each experimental or training session by having monkeys maintain fixation at known visual angles.

The animals were trained on three behavioral tasks. Each trial began with the appearance of a fixation point (FP, $0.5^{\circ}$ square) at the center of the screen. Monkeys were required to maintain fixation for 800-1200 msec to proceed with the trial. Different stimuli were presented in different tasks (see below), and monkeys made eye movements in response to the FP offset. Correct performance was reinforced with a drop of liquid reward at the end of each trial. Different tasks were presented in random order within a block that usually consisted of 12 or 16 trials (four or six covert tracking trials and four or six single target trials, two memory-guided saccade trials and two smooth pursuit trials in opposite directions). The animals discriminated the tasks based on visual stimuli presented during fixation and could make a correct response as the FP disappeared.

\section{Covert Tracking Task}

After the initial fixation interval, two visual stimuli $\left(2^{\circ}\right.$ white circles, $17.1 \mathrm{~cd} / \mathrm{m}^{2}$ ) were presented (Figure 1A, left). Their initial locations were defined in polar coordinates, with $10^{\circ}$ eccentricity, and angles were chosen randomly from $0^{\circ}$ to $350^{\circ}$ ( $10^{\circ}$ increments, measured from rightward). The separation of polar angles between the two stimuli was either $60^{\circ}, 120^{\circ}, 180^{\circ}, 240^{\circ}$, or $300^{\circ}$. One object briefly changed its color (red, $300 \mathrm{msec}$ ) and served as the target, whereas the other remained white throughout the trial and served as the distractor. Except for this cue period, the two objects were visually identical. Following a 300-msec delay, both objects moved along straight paths at $20^{\circ} / \mathrm{sec}$ in different directions and bounced against the sides of $40^{\circ}$ square (motion period, $3000 \mathrm{msec}$ ). Because the stimulus diameter was $2^{\circ}$, the center of each object moved within a $38^{\circ}$ square. The initial motion direction for each object was chosen randomly from $0^{\circ}$ to $330^{\circ}$ ( $30^{\circ}$ increments), but the separation of initial motion directions between objects was set within a range from $60^{\circ}$ to $300^{\circ}$. Monkeys were required to keep their eyes within $5^{\circ}$ of the FP throughout the motion and the following delay (1000 msec) periods and then to make a saccade to the target within $400 \mathrm{msec}$ after the FP offset. When the target position at the end of the motion period was $<5^{\circ}$ from the FP or the distance between the two objects was $<5^{\circ}$, the motion interval was extended until neither condition was fulfilled.

Once a monkey successfully performed a covert tracking trial, three trial conditions were conducted to obtain control data and to balance the object trajectories during recording sessions. First, each covert tracking trial was immediately followed by a single target trial in which only the target appeared and moved along the same path as in the previous trial. Second, in the subsequent covert tracking trial, the trajectories of the target and the distractor were interchanged. Third, in the following two covert tracking trials, the trajectories of the target and the distractor were rotated by $180^{\circ}$. Thus, once monkeys completed a covert tracking trial correctly, the object trajectories in the following three covert tracking trials and four single target trials were determined automatically.

During training sessions, we presented up to four (monkey L) or five (monkey F) distractors with a single target. Neuronal data were obtained only after monkeys were well trained and were able to choose the target correctly at a rate well above chance (Figure 2 ). For the experiment illustrated in Figure 8, we used a modified covert tracking task (Figure 1A, right). In this task, a second, green $\left(19.4 \mathrm{~cd} / \mathrm{m}^{2}\right)$ distractor was also presented along with the original white distractor and the target. The second distractor had the same shape and size $\left(2^{\circ}\right)$ as the other objects; only its color differed. As in the original covert tracking task, each modified covert tracking trial was followed by a single target trial, and the trajectories of all three objects were interchanged in the following trials. 
Figure 1. Behavioral paradigms employed in the present experiment. (A) In the covert tracking task (left), two visual stimuli were presented during monkeys fixated on the central FP. One object briefly altered its color and served as a target. Following a short delay, two objects randomly moved for $>3 \mathrm{sec}$. Monkeys were rewarded for making a saccade to the target after the FP disappeared. Because the target and the other object (distractor) were identical in color and shape except for the cue period, monkeys needed to keep track of the target throughout the motion period. Once a monkey successfully performed a covert tracking trial, three trial conditions followed. First, each covert tracking trial was immediately followed by a single target trial in which only the target appeared and moved along the same path as in the previous trial. Second, in the subsequent covert tracking trial, the trajectories of the target and the distractor were interchanged. Third, in the following two covert tracking trials, the trajectories of the target and the distractor were rotated by $180^{\circ}$. In the modified covert tracking task (right), an additional green distractor was presented along with the original white distractor and target. (B) In the memory-guided saccade task, a visual cue was presented during central fixation. Monkeys were rewarded for making a saccade to the cued location after the FP disappeared.

\section{Memory-guided Saccade Task}

During central fixation, a visual cue $\left(1^{\circ}\right.$ square $)$ was presented briefly $(200 \mathrm{msec})$ at $12^{\circ}$ eccentricity. Monkeys remembered its location and maintained fixation for an additional $2000 \mathrm{msec}$. Once the FP disappeared, monkeys were required to make a saccade to the previously cued location within 400 msec.

\section{Smooth Pursuit Task}

Because the main task in this study involved covert tracking, we also included smooth pursuit task to examine neuronal activity during overt tracking. After a 1200-msec fixation interval, the FP was replaced with a moving target ( $1^{\circ}$ square), which appeared $3^{\circ}$ eccentrically and moved toward the location of initial fixation at $20^{\circ} / \mathrm{sec}$ (Rashbass stimuli). Following the excursion for $1000 \mathrm{msec}$, the target stopped and remained visible for an additional $800 \mathrm{msec}$. Monkeys were required to maintain eye position within $4^{\circ}$ of the target throughout the motion and fixation periods. For the activity during pursuit, the firing rates during the baseline period (300 msec before target motion) and the motion period (1000 msec) were compared using a one-way ANOVA. Among the target-selective-type neurons examined in the pursuit task, 31\% (49/158) exhibited elevated firing rates during pursuit, 17\% (27/158) exhibited reduced firing rates, and the others exhibited no modulation. In contrast, only a minority of the Distractor-selective-type neurons exhibited firing modulation during pursuit (enhancement: 4/41, 10\%, suppression: 6/41, 15\%). We did not perform any further analysis for neuronal activity during pursuit in this study.

\section{Physiological Procedures}

Neuronal activity was recorded through tungsten electrodes (FHC, Inc., [Bowdoin, ME] or Alpha Omega Engineering [Nazareth, Israel]) lowered into the PFC though a 23-gauge

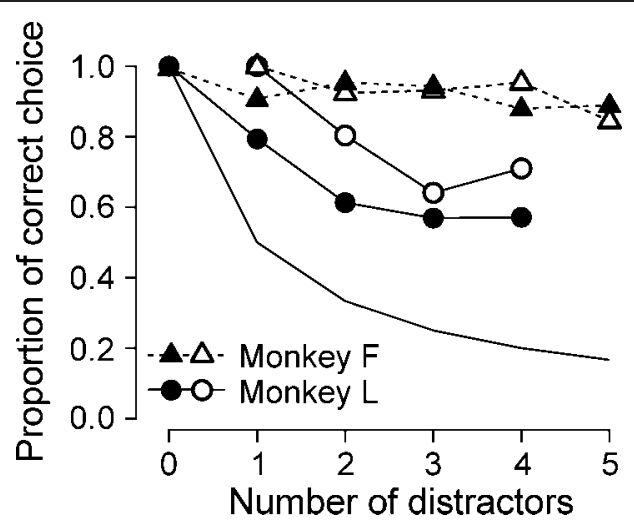

Figure 2. Behavioral data. For each monkey, proportions of correct choice are plotted as a function of the number of distractors for trials with (open symbols) or without (filled symbols) a green, explicit distractor. Note that all data points were well above the chance level (thin black lines). 
guide tube using a micromanipulator (MO-97S; Narishige, Tokyo, Japan). The location of electrode penetration was adjusted using the X-Y stage attached on top of the cylinder. When searching for task-related neurons, monkeys performed an easy version of the covert tracking task, in which the target remained red throughout the trial, and the eye movement tasks. Once task-related neuron was isolated, the task was switched to the ordinary covert tracking task described above. Signals obtained from the electrodes were amplified (Model 1800; A-M Systems, Sequim, WA), filtered (Model 3625; NF Co., Tokyo, Japan), and monitored on-line using oscilloscopes and an audio device. The waveforms of a single neuron were isolated using a real-time spike sorter with template-matching algorithms (MSD; Alpha Omega Engineering). Occurrences of action potentials were time stamped (sampling rate: $1 \mathrm{kHz}$ ) and saved in files with the data of eye movements and stimulus locations during experiments. In some penetrations, we also delivered electrical stimulation $(0.2 \mathrm{msec}$ biphasic 34 pulses at $333 \mathrm{~Hz}$, $\leq 50 \mu \mathrm{A})$ to locate the FEF.

A postmortem examination of recording sites was performed in monkey $\mathrm{F}$ (Figure 3A). At the end of the experiments, the animal was deeply anesthetized with sodium pentobarbital (>50 mg/kg, ip), and several landmark pins were penetrated at known coordinates. The animal was then perfused with $0.1 \mathrm{M}$ phosphate buffer followed by $3.5 \%$ formalin. The location of each task-related neuron was reconstructed according to the coordinates of electrode penetrations relative to the landmark pins.

\section{Data Analysis}

Data were analyzed off-line using Matlab (Mathworks, Natick, MA). We considered only neurons exhibiting a sig- nificant firing modulation depending on object locations. For the initial screening, pseudocolor maps of instantaneous firing rate were constructed for each moving object, and the existence of firing modulations was detected visually. Then the variance of firing rates in a pseudocolor map was statistically verified for each neuron. To estimate the intrinsic variance of neuronal activity, a correspondence between spike data and object trajectories were shuffled and a pseudocolor map was reconstructed. We repeated this shuffling procedure 1000 times to obtain the distribution of eventual variance. Neurons exhibiting a greater variance of firing rates $(p<.05)$ for one or both objects were included for further analyses (266/311, 86\%).

Among 266 neurons that modulated activity in the covert tracking tasks, 209 were also tested in the memory-guided saccade task. For the memory-guided saccade task, the firing rates during the following intervals were measured: (1) a 300msec interval immediately before the cue onset (baseline period), (2) a 200-msec interval starting from 100 msec after the cue onset (visual response period), (3) a 300-msec interval immediately before the FP offset (delay period), and (4) a 150-msec interval starting from $100 \mathrm{msec}$ before the saccade onset (movement period). The firing rates during these periods were compared using a one-way factorial ANOVA. Neurons were classified as having visual, delay, or motor responses when post hoc multiple comparisons (Scheffé's test) revealed a significant firing modulation during the corresponding intervals compared with baseline activity $(p<$ $.05)$. Table 1 summarizes the number of those neurons.

\section{RESULTS}

We examined single neuron activities in the lateral PFC when monkeys covertly tracked one of identical, moving
Figure 3. Recording sites. (A) For monkey $\mathrm{F}$, recording sites were reconstructed according to the coordinates of electrode penetrations relative to the landmark pins located at known coordinates. The size of each symbol (red circle, target-selective type; blue square, distractor-selective type) indicates the number of neurons recorded from each penetration. The X's indicate the sites where electrical microstimulation $(\leq 50 \mu \mathrm{A})$ evoked saccades. PS, principal sulcus. AS, arcuate sulcus. (B) Recording sites in monkey L. Because this monkey is still in use for another project, the relative location of electrode penetrations are shown. Although the recordings were made from the right frontal cortex, the coordinates are flipped to the left hemisphere to allow for comparison with monkey $\mathrm{F}$.

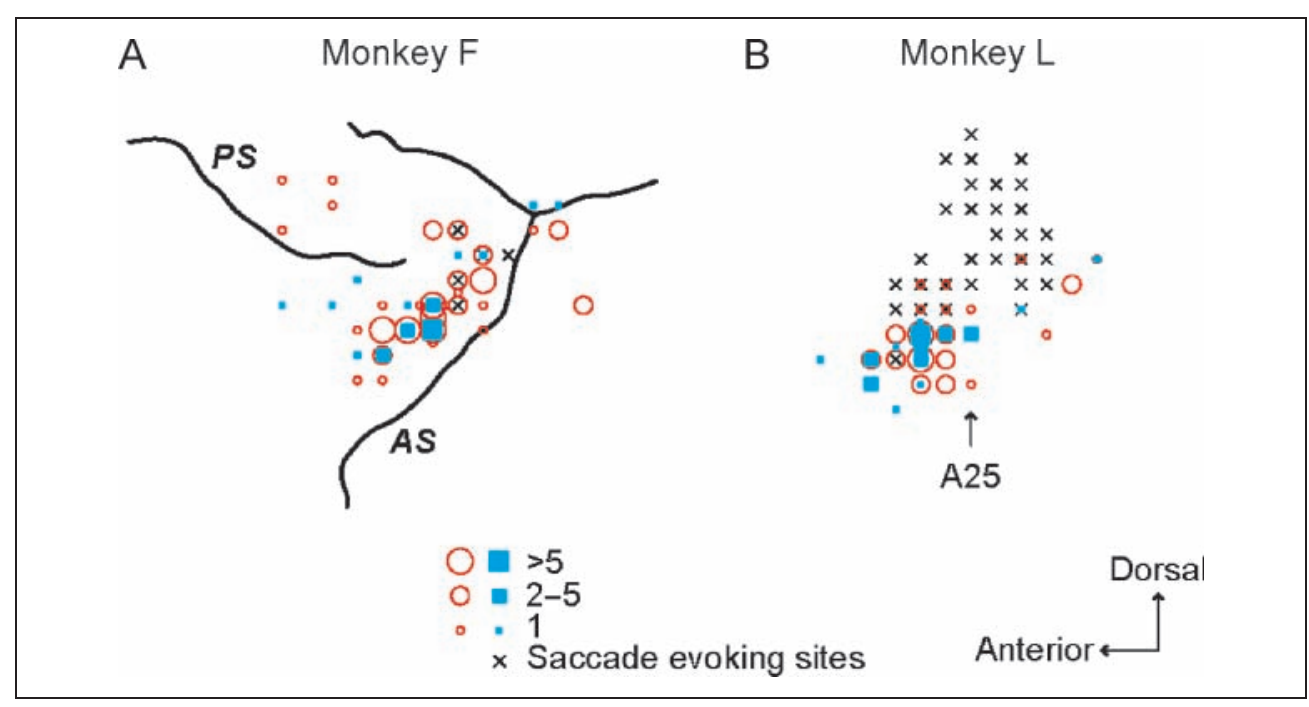


Table 1. Summary of Neuronal Activity during the Memory-guided Saccade Task

\begin{tabular}{|c|c|c|c|c|c|c|c|}
\hline \multicolumn{3}{|c|}{ Response during Covert Tracking } & \multirow[b]{2}{*}{ Number of Neurons Tested } & \multicolumn{4}{|c|}{$\begin{array}{l}\text { Response during Memory Saccade } \\
\qquad(n=209)\end{array}$} \\
\hline Type of Response & & RF Location & & Visual & Delay & Motor & None \\
\hline \multirow[t]{3}{*}{ Target-selective ( $n=158$ ) } & \multirow[t]{3}{*}{$\operatorname{Tg}$} & Ipsi & 43 & $19(2)$ & $15(3)$ & $15(3)$ & 14 \\
\hline & & Contra & 89 & $63(11)$ & $30(9)$ & $40(9)$ & 16 \\
\hline & & Vertical & 26 & $13(2)$ & $4(0)$ & $10(0)$ & 10 \\
\hline \multirow[t]{3}{*}{ Distractor-selective $(n=41)$} & \multirow[t]{3}{*}{ Dis } & Ipsi & 17 & $3(1)$ & $5(1)$ & $4(1)$ & 9 \\
\hline & & Contra & 20 & $13(0)$ & $4(1)$ & $9(0)$ & 3 \\
\hline & & Vertical & 4 & $2(1)$ & $0(0)$ & $3(1)$ & 0 \\
\hline \multirow[t]{3}{*}{ Nonselective $(n=10)$} & \multirow[t]{3}{*}{$\operatorname{Tg}$} & Ipsi & 1 & $0(0)$ & $1(0)$ & $1(0)$ & 0 \\
\hline & & Contra & 6 & $5(0)$ & $0(0)$ & $1(0)$ & 1 \\
\hline & & Vertical & 3 & $2(0)$ & $0(0)$ & $2(0)$ & 1 \\
\hline
\end{tabular}

Number in parentheses indicates the number of neurons showing a significant suppression.

objects without eye movements (Figure 1A, left). In the covert tracking task, two identical objects moved randomly in a visual field while monkeys maintained central fixation. Because monkeys had to make a saccade to the object that was cued at the beginning of each trial (target), they needed to keep track of the target throughout the motion period. Figure 2 plots the proportion of correct choice as a function of the number of distractors for both monkeys. Because the distractors were visually identical to the target, the monkeys occasionally missed the target, and performance declined as the number of distractors increased. Although the animals were trained to choose a target among up to four or five distractors at a rate well above the level expected by chance (Figure 2, data with filled symbols), only a single distractor was presented during the recording sessions. Both monkeys performed very well during the recording sessions (mean \pm $S D$ of the rate of correct choice: monkey F, $90.7 \pm 7.2 \%$; monkey L, $97.2 \pm 2.6 \%$ ).

Our recording sites were located in the anterior lip of the inferior limb of the arcuate sulcus (Figure 3), where the previous study referred to as pre-FEF (Hasegawa, Peterson, \& Goldberg, 2004). We found many neurons that modulated the activity during the covert tracking task, as an example shown in Figure 4A. Although this neuron elevated the activity as the visual stimuli were presented, the firing varied from trial to trial because of the randomized object trajectories. To assess neuronal modulation depending on object locations during the motion period, the instantaneous firing rate was calculated as a function of each object location. For this analysis, each object locations at $60 \mathrm{msec}$ before the occurrence of action potentials were plotted separately on different panels (Figure 4C, top). We chose 60 msec according to the previous studies that examined the visual response latency in the FEF (Pouget, Emeric, Stuphorn, Reis, \& Schall, 2005; Thompson,
Hanes, Bichot, \& Schall, 1996; Bruce \& Goldberg, 1985). Then the number of action potentials was divided by the duration of object presentation to obtain the firing rate for each pixel $\left(0.5^{\circ}\right.$ square $)$. The data were filtered using a 2-D Gaussian kernel $\left(\sigma=2.5^{\circ}, 20^{\circ}\right.$ square, $0.5^{\circ}$ resolution) and were presented as pseudocolor maps (Figure 4C, bottom). Because the same spike data were used to compute the maps for the target and distractor, any difference in the pseudocolor maps was attributed to the different meaning of each object in the task. Furthermore, to balance the trajectories of the target and distractor, these objects moved along the same path in successive covert tracking trials with the inverse assignment of object meaning between the trials (colored dots on black lines in Figure 4C; see Methods). This neuron exhibited a strong response when the target passed through its receptive field (RF) in the top right visual field (Figure 4C, bottom left), but exhibited only a weak response when the distractor was presented in the RF (bottom right). Figure $4 \mathrm{~B}$ plots the activity of the same neuron in the memory-guided saccade task, exhibiting visual, delay, and presaccade activity for the tasks with cues in the top right visual field. Like this example, most neurons with firing modulation during the covert tracking task were also responsive in the conventional memory-guided saccade task (Table 1).

Because the monkeys were required to maintain eye position within $5^{\circ}$ of the central FP during covert tracking, the neuronal response maps computed from retinal locations of object images were qualitatively the same as those computed from spatial locations on the screen (Figure 4D, right). The left of Figure 4D plots eye position during the entire motion period in the same recording session, and shows that the deviation of eye position from the FP was much less than the window size (mean $\pm S D, 0.95^{\circ} \pm 0.53^{\circ}$ ). For all recording sessions, 

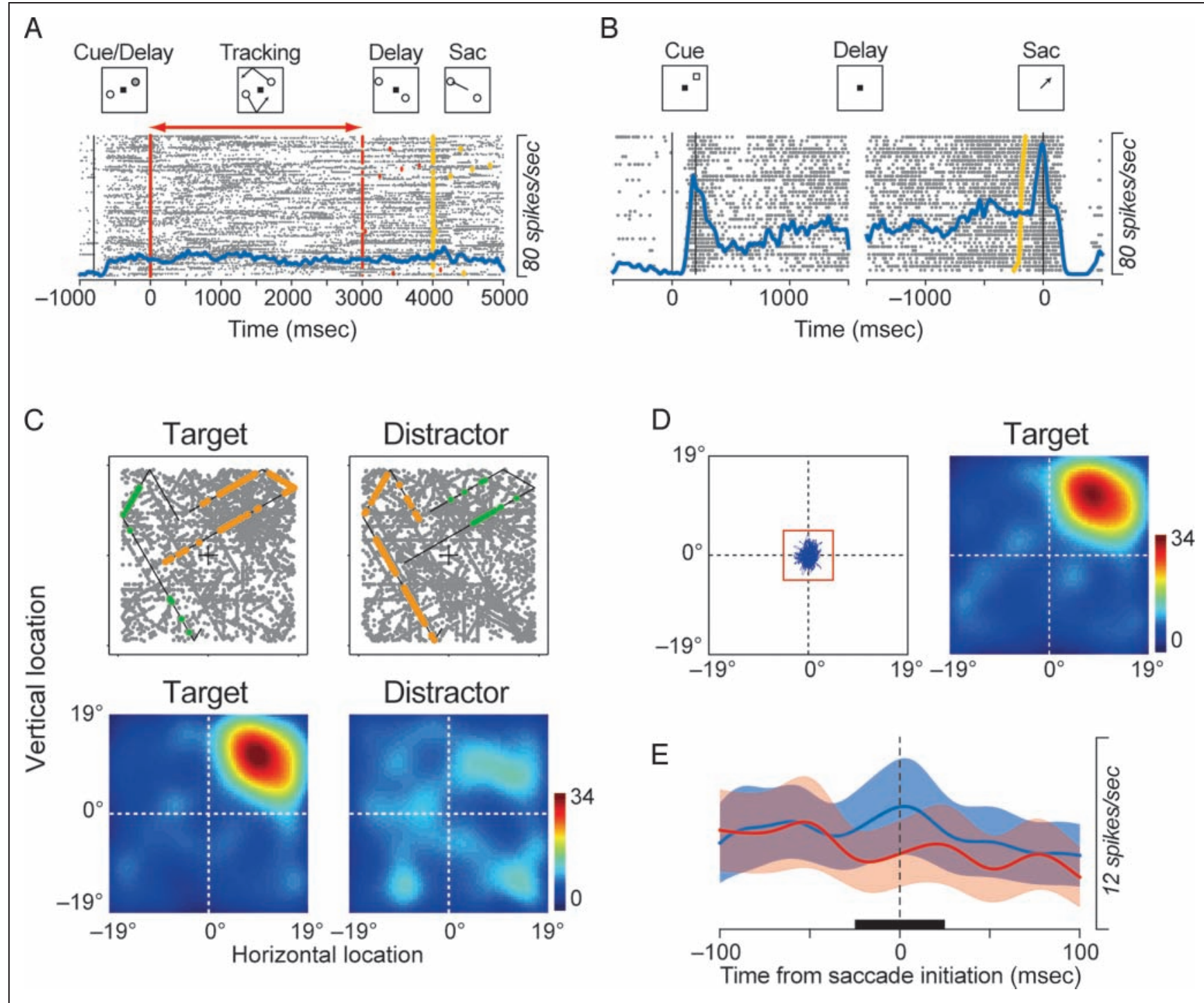

Figure 4. Response of an example neuron. (A) Activity during the covert tracking task. In raster plot, each dot represents the time of action potential and each row represents a single trial. Data are aligned with the onset of object motion (red vertical line). The subsequent red dots indicate the termination of object motion. Blue trace shows spike density for the associated rasters $(\sigma=15 \mathrm{msec})$. Black vertical line and yellow symbols indicate the onset and offset of the FP, respectively. Because the objects moved randomly during motion period (indicated by red arrows), neuronal activity was also random. (B) Activity during the memory-guided saccade task. Data are aligned with either the cue onset (left column) or the initiation of saccades (right column). Trials are sorted according to saccade latencies. Yellow symbols indicate the FP offset. (C) Two dimensional plots of instantaneous neuronal activity during covert tracking. Gray dots in the top panels indicate locations of the target (left) or the distractor (right) $60 \mathrm{msec}$ before the occurrence of action potentials. Note that the number of gray dots on each panel is the same. Colored dots on black lines show the data for two sample successive trials, in which the trajectories of the target and distractor were interchanged. The pseudocolor maps indicate instantaneous firing rates for different objects (bottom). Calibration bar indicates firing rates (spikes/sec). (D) Left panel plots eye positions during the entire motion period in the same recording session as shown in A and C. Red square indicates $5^{\circ}$ fixation window. Right panel shows the response map based on the locations of retinal target images, computed by subtracting eye position from target locations on the screen. Calibration bar is the same as in B. (E) Time course of neuronal activity around the time of micro-saccades toward (red line) or away from (blue line) the RF. Shaded areas represent $95 \%$ confidence intervals. Black bar indicates the 50-msec interval for statistical comparison.

the magnitude of eye deviation averaged $0.90^{\circ} \pm 0.16^{\circ}$ and $0.59^{\circ} \pm 0.08^{\circ}$ for monkeys F and L, respectively. Furthermore, neuronal activity around the time of microsaccades $( \pm 25 \mathrm{msec}$ ) toward (Figure 4E, red trace) or away from (blue trace) the RF during covert tracking was not significantly different for both the example neuron shown in Figure 4 (unpaired $t$ test, $p>.05$ ) and the population of 266 neurons (paired $t$ test, $p>.05$ ). Given that neurons in many cortical areas represent visual space in an eye-centered coordinate frame (Golomb, Chun, \& Mazer, 2008; Cohen \& Andersen, 2002), the response maps were computed in retinal coordinates by subtracting eye positions from the actual stimulus locations for the subsequent analyses and in the remaining figures. 


\section{Different Types of Neuronal Activity during Covert Tracking}

We recorded from 167 neurons exhibiting a significant firing modulation by object locations during covert tracking. To quantify the magnitude of firing modulation depending on object locations, we measured pixel-by-pixel variance of instantaneous firing rates for each pseudocolor map. Because the variance in a given map increases as neuronal activity depends more on the object locations, we classified neurons based on the magnitude of variance for each object. "Target-selective type" neurons $(130 / 167,78 \%)$ exhibited a greater variance of firing rates for different target locations than for distractor locations ( $F$ test, $p<.05)$. "Distractor-selective type" neurons (30/167, $18 \%)$ exhibited a greater variance for distractor locations than for target locations $(p<.05)$. "Nonselective type" neurons $(7 / 167,4 \%)$ exhibited no preference for either object $(p>.05)$, while they modulated the activity depending on the location of each object (Methods). For further quantification, we also computed the maximal firing modulation defined as the difference between maximal and minimal activity calculated for every $15^{\circ}$ circular areas in each response map ( $0.5^{\circ}$ steps). The maximal firing modulation correlated well with the variance $(r=.88, p<$ $.0001 ; 167$ neurons $\times 2$ objects). We also confirmed that the maximal firing modulation for the target was significantly larger than that for the distractor in Target-selective type neurons (paired $t$ test, $p<.05$ ), whereas the firing modulation for the target was significantly less than that for the distractor in distractor-selective type neurons (paired $t$ test, $p<.05$ ).

Figure 5 shows three different types of representative neurons. The nonselective type neuron (Figure 5A) exhibited enhanced activity for any object presented in the $\mathrm{RF}$, irrespective of object meaning in the task, which suggested that these neurons reflected only physical locations of visual stimuli. On the other hand, the majority of neurons exhibited target-selective responses similar to the neuron shown in Figure 5C, which responded more to the target than to the distractor. Both the nonselective type and target-selective type neurons typically exhibited

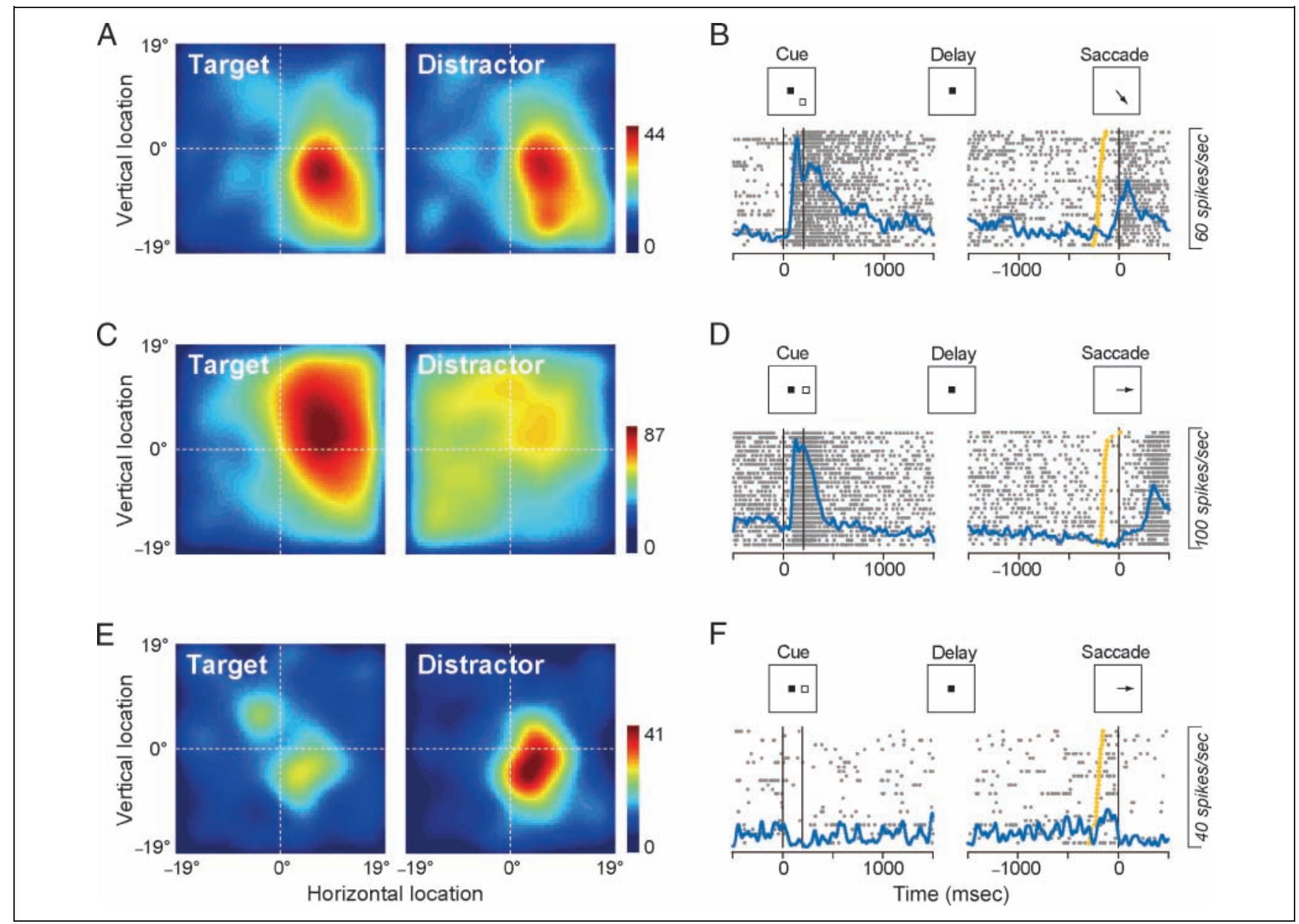

Figure 5. Examples of three different types of neurons. Each row illustrates the data from a single neuron. The neuron type was determined according to the variance of instantaneous neuronal firing. (A and B) The response maps during the covert tracking task and the activity during the memory-guided saccade task, respectively, for a non-selective type neuron. (C and D) Target-selective type neuron. (E and F) Distractor-selective type neuron. Although this neuron exhibited virtually no firing modulation in the memory-guided saccade trials, many other distractor-selective type neurons showed a significant modulation (Table 1). 
strong visual responses in memory-guided saccade trials presented in the RF (Table 1; Figure 5B and D). Neuronal modulation selective to the target suggests that the brain represents the target location at every moment during covert tracking, and signal highlighting the most relevant location is dynamically updated in the population of cortical neurons.

We also found neurons that exhibited greater firing modulation for the distractor than the target. As shown in Figure 5E, these neurons responded strongly to the distractor but displayed only a weak response, if any, to the target. Because the trajectories of the target and distractor were chosen randomly and independently, the selective response to the distractor could not be explained by simple suppression from the target-selective type neurons. In the memory-guided saccade trials, the distractor-selective type neurons typically exhibited transient responses to visual cues presented in the RF (Table 1, see an example in Figure 8B), although the neuron shown in Figure $5 \mathrm{E}$ had a parafoveal RF and failed to respond to visual stimulus presented at $12^{\circ}$ eccentricity (Figure $5 \mathrm{~F}$ ). Neuronal modulation selective for the distractor suggests that the brain also continuously monitors the distractor during covert object tracking.

Although most neurons exhibited increased activity when the nonpreferred object came into the RF, some exhibited a slight suppression. Figure 6 shows two such examples. The target-selective type neuron shown in Figure $6 \mathrm{~A}$ had its RF in the top right visual field (top left) but exhibited decreased activity for the distractor presented in the RF (top right). Similarly, the distractorselective type neuron shown in Figure $6 \mathrm{~B}$ had its RF in the right visual field (top right) but exhibited decreased activity for the target in the RF (top left). For both neurons, the activity for the nonpreferred object in the RF (defined as the $15^{\circ}$ circular area showing maximal activity across both maps) was significantly less than the mean of the entire map for the nonpreferred object ( $t$ test, $p<$ $.05)$. Among 130 target-selective type and 30 distractorselective type neurons, 34 (26\%) and 7 (23\%) exhibited a significant suppression. Because these neurons displayed maximal activity for a specific combination of the target and distractor locations, they appeared to carry information of both objects. Indeed, the neuron shown in Figure 6A exhibited maximal activity when the target was located in the top visual field, and at the same time, the distractor was located in the bottom visual field (Figure 6A, bottom). The neuron shown in Figure 6B exhibited maximal activity for the combination of the target in the left visual field and the distractor in the right visual field (Figure 6B, bottom). Given that the firing modulation depending on the meaning of the object in the RF was maximal for this type of neurons, these signals could be utilized to internally contrast object identities during covert tracking.

Although it is plausible that the distractor-selective modulation was related to the selective suppression of

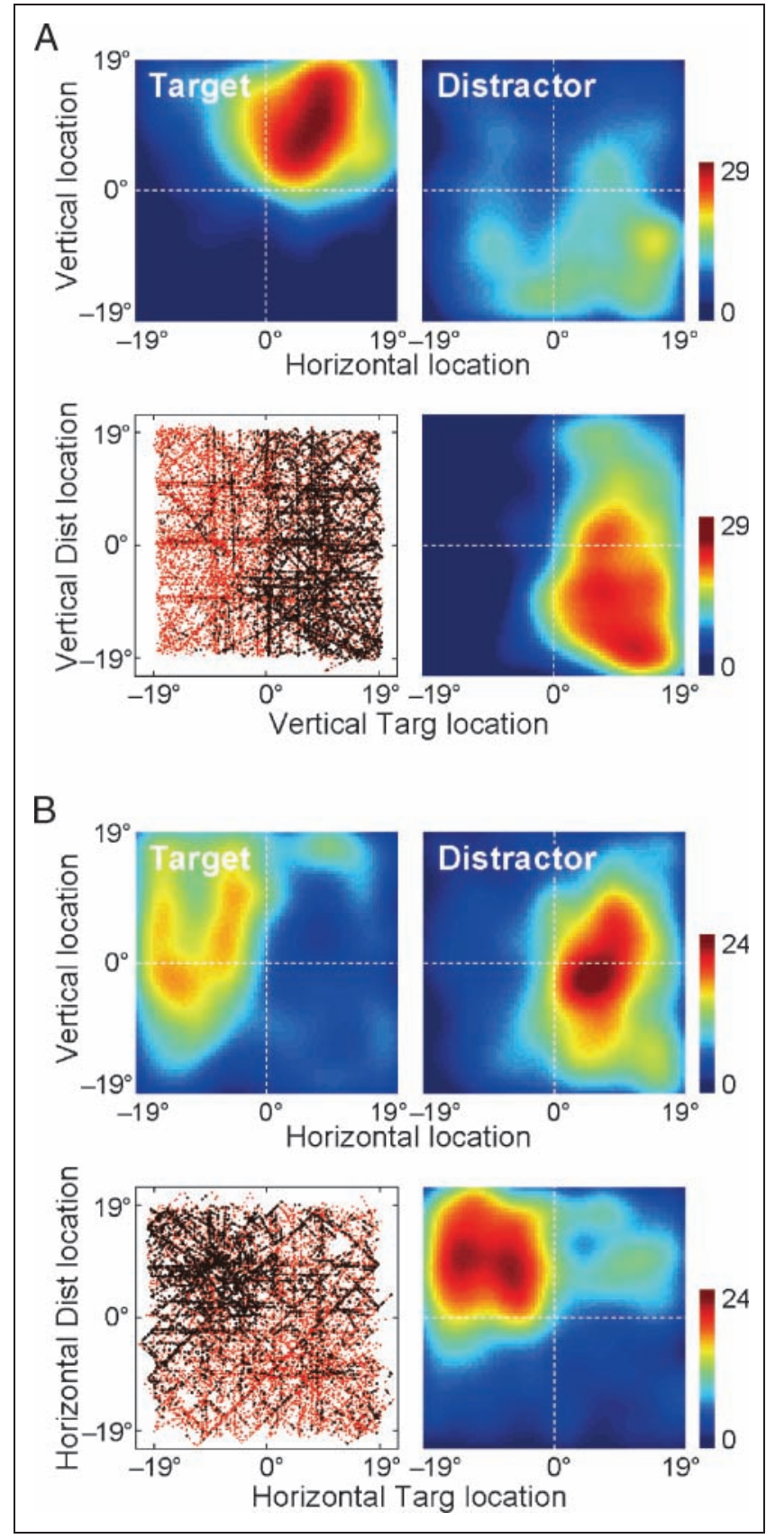

Figure 6. Neurons responding to a specific combination of object locations. (A) Target-selective type neuron. Response maps for the target (top left) and the distractor (top right). Red dotted lines in the bottom left panel plot vertical distractor location as a function of vertical target location throughout the motion period. Black dots indicate the data 60 msec before the occurrence of action potentials. The pseudocolor map (bottom right) indicates instantaneous firing rates computed from the data in bottom left. (B) Distractor-selective type neuron. Response maps for the target (top left) and the distractor (top right). Neuronal modulation for combinations of horizontal object locations are presented in the bottom. Note that all possible combinations of object locations were thoroughly tested (red lines in A and B).

visual processing for the distractor reported previously, one might argue that it could also be related to attentional enhancement of the distractor. According to the biased competition theory (Desimone, 1998; Desimone 
\& Duncan, 1995), attention is automatically guided toward items that match current goals. Because the distractor was physically identical to the target in this study, the attention might be automatically biased toward the distractor. If this is the case, the enhancement of the response to the distractor would lead to an erroneous target choice. However, we found opposite results as examined in the data in error trials. For example, Figure 7 compares the activity for the neuron shown in Figure 6B between trials with correct (Figure 7A) and erroneous (Figure 7B) target choice. In error trials, the neuron did not show enhanced activity for the distractor in the RF but instead exhibited the opposite pattern of the responses to the target and distractor. These results imply that the distractor-selective modulation might reflect active suppression rather than attentional enhancement of the distractor.

\section{Absence of Neuronal Modulation for an Explicit Distractor}

Neuronal activity during covert object tracking with a single white distractor, as described above, suggests a role for the pre-FEF in object specification and discrimination. However, if the distractors explicitly differ from the target, for example, in color, they could be easily ignored and topdown signals for distractor monitoring might not be necessary. In fact, previous psychophysical studies have shown that visual processing at distractor location is actively suppressed only when the distractor is similar to the target (Thomas \& Lleras, 2009; Pylyshyn et al., 2008; Muller \& von Muhlenen, 2000; Klein, 1988). To explore neuronal correlates, activity of another 99 neurons was examined while monkeys performed a modified covert tracking task (Figure 1A, right). In this task, an additional green distractor was presented along with the original white distractor and target. The second, green distractor was salient but was never a target, so the monkeys could easily ignore it. Indeed, the relationship between the rate of correct choice and the number of distractors shifted rightward in the modified covert tracking task (Figure 2, open symbols), indicating that the green distractor did not interfere with the performance.

As in the first experiment, we measured the instantaneous firing rates for each of the three objects. According to the same classification procedure that compared the variances of neuronal activity in the response maps, many neurons again exhibited target-selective response (65/99, $66 \%)$, whereas the others exhibited either distractorselective $(26 / 99,26 \%)$ or nonselective (8/99, 8\%) responses. As shown in Figure 8A and B, both the target-selective type and the distractor-selective type neurons exhibited only a weak response to the green, explicit distractor. The absence of responses to the green distractor was observed across the neuronal population (Figure 8C). Of the 35 neurons exhibiting a preference for either distractor relative to the target, 27 (77\%) responded more to the original, implicit distractor than to the green, explicit distractor. These results suggest that neurons in the pre-FEF are likely involved in specific marking of the implicit distractor, which is identical to the target and therefore requires additional
Figure 7. Activity of a distractor-selective type neuron predicted subsequent target choice. (A) Data for the neuron shown in Figure 6B in correct trials. Red and blue dotted lines (left) indicate the retinal locations of the target and distractor images during covert tracking, respectively. Each black dot represents the data 60 msec before the occurrence of an action potential. The right panel plots the instantaneous firing rates for each combination of horizontal object locations, demonstrating that the neuron responded only when the target was located in the left visual field and the distractor was in the right visual field. (B) Data in error trials. Note that the locations of response fields for the target and distractor were reversed.

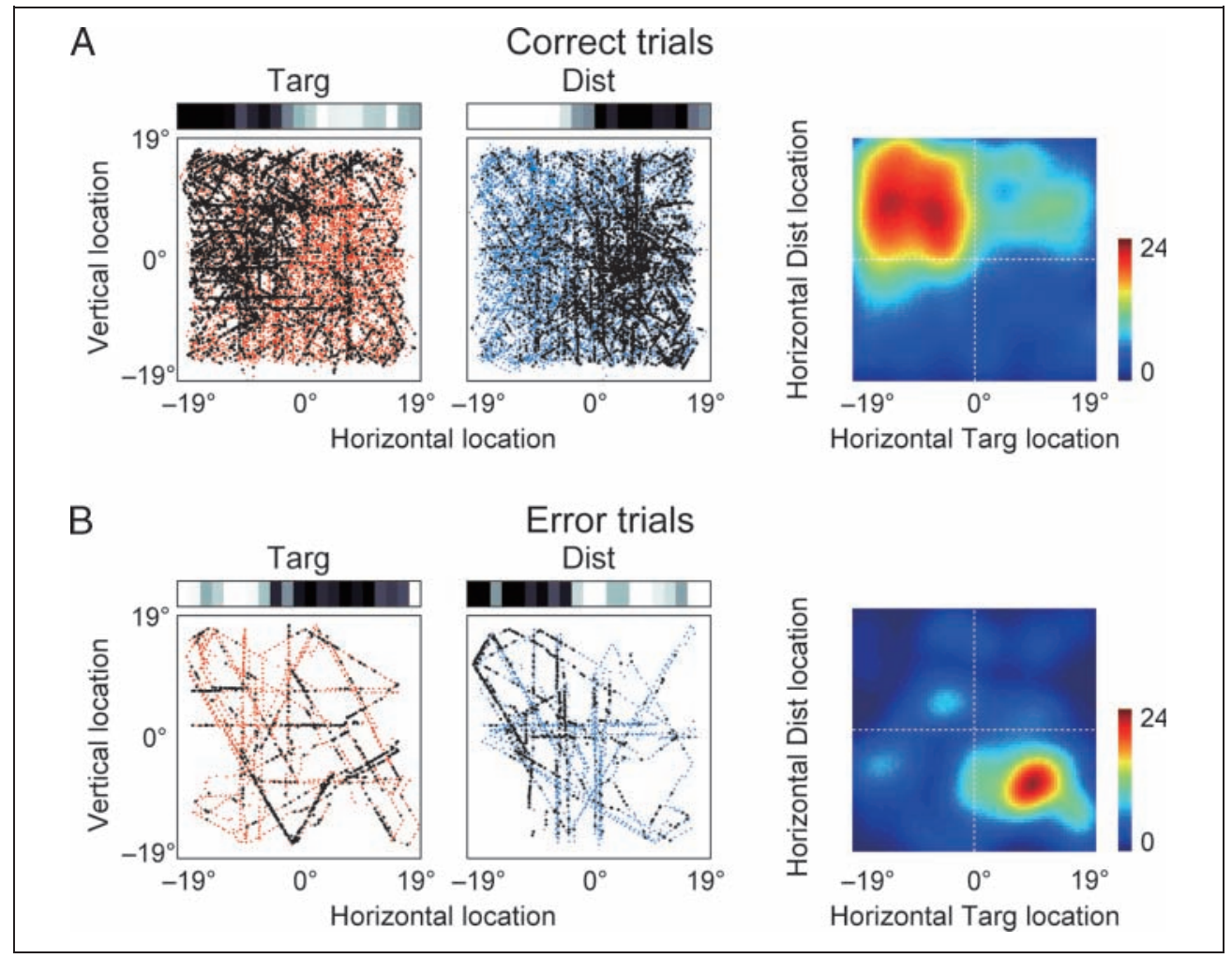




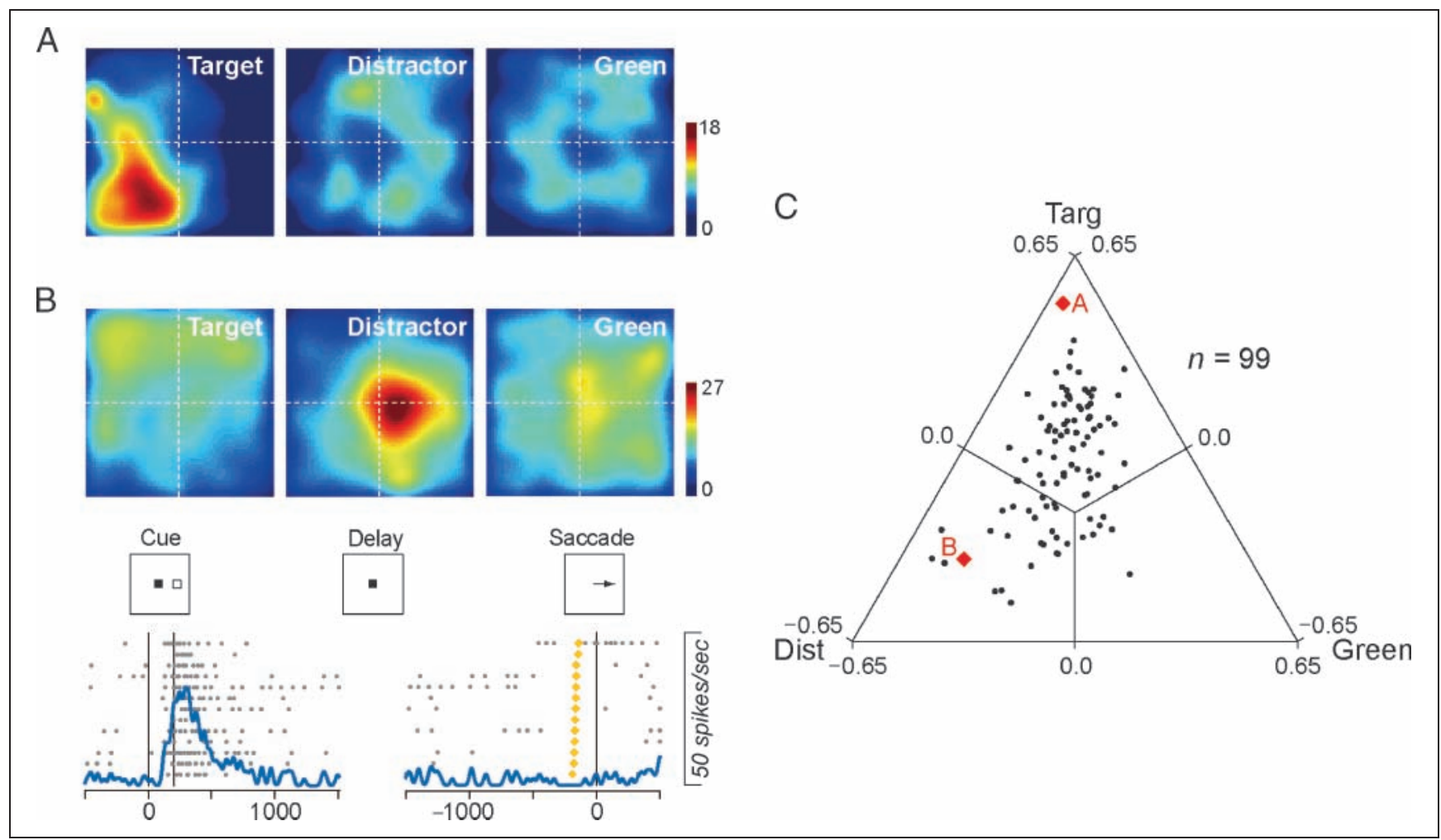

Figure 8. Absence of the response to an explicit distractor. (A) Target-selective type neuron. (B) Distractor-selective type neuron. Responses to different objects in the modified covert tracking task are plotted separately on different panels. Note that both neurons responded only weakly to the green, explicit distractor (right). The neuron in B showed a phasic visual response during memory-guided saccade trials (bottom). (C) Comparison of the firing modulation between the objects for individual neurons. The maximal firing modulation (Mod) was measured for each object and was normalized so that $\operatorname{Mod}_{\mathrm{tg}}+\operatorname{Mod}_{\mathrm{dis}}+\operatorname{Mod}_{\text {green }}=1$. The horizontal axis of the triangular plot corresponds to Mod ${ }_{\text {green }}-$ Mod $_{\mathrm{dis}}$, whereas the vertical axis corresponds to $\sqrt{ } 3 \times \operatorname{Mod}_{\mathrm{tg}}$. Red diamonds indicate the data for the neurons shown in $\mathrm{A}$ and $\mathrm{B}$.

top-down signals for discrimination. The brain might expend extra effort to track the distractor only when it is indistinguishable from the target.

\section{Time Course of the Population Activity}

We next examined the dynamics of spatially specific firing modulations described so far. For each neuron, the RF was defined as a $15^{\circ}$ circular area giving a maximal firing rate across the pseudocolor maps for the target and the distractor. We then extracted the data around the time when each object crossed the RF border $( \pm 300 \mathrm{msec})$ and remained inside or outside the $\mathrm{RF}$ for $\geq 300 \mathrm{msec}$. The firing rates during the 600-msec interval for each direction of motion (i.e., toward or away from the RF) were computed for individual neurons, and the data were normalized by baseline activity (mean firing rate during the entire motion period). Figure 9 shows the population activity for 195 target-selective type neurons (monkey F, $n=146$; monkey L, $n=49$ ) and 56 distractor-selective type neurons (monkey F, $n=27$; monkey L, $n=29$ ) recorded during the (modified) covert tracking trials. Figure 9A plots the time courses of population activity for the target-selective type neurons, which elevated the firing rate for the target coming into the $\mathrm{RF}$ (orange trace) but not for the distractor (green trace). In contrast, the distractor-selective type neurons increased their activity for the distractor in the RF but not for the target (Figure 9B). Again, because the object trajectories were exactly identical in two successive covert tracking trials, any difference in activity should be attributed to the difference in object meaning in the task. In both types of neurons, the preference for a specific object was most conspicuous when the object was presented in the RF.

For both types of neurons, the object preference was consistently observed when the object was located in the $\mathrm{RF}$. To compare the properties of neuronal activity between different time windows during the 3.0-sec motion period, data were separately analyzed for four 750 -msec intervals starting from the motion onset. For each neuron, we measured the average firing rate when each object was presented within the RF. Then the neuronal discriminability was quantified by computing the receiver operating characteristic (ROC) values that compared the response to the target with that to the distractor. The ROC value close to zero or unity indicates a strong preference for the distractor or target, respectively. The ROC value for the target-selective type neurons (red line in Figure 9C) was approximately 0.75 and remained unchanged throughout the motion period, indicating that these neurons consistently responded 
to the target. Similarly, the ROC value for the distractorselective type neurons was approximately 0.25 throughout the motion period (blue line in Figure 9C), indicating that these neurons consistently detected the distractor. Thus, the object meanings were continuously represented in the two populations of neurons in the pre-FEF during covert tracking, with a remarkable consistency.

\section{Spike Waveform Analysis}

Recent studies have demonstrated that the width of action potentials correlates with the properties of attentional modulation in V4 neurons (Anderson, Mitchell, \& Reynolds, 2011; Mitchell et al., 2007). For a limited number of pre-FEF neurons, we saved the data of spike waveforms for off-line analysis and measured the width of action potentials (the time between the trough and the peak of

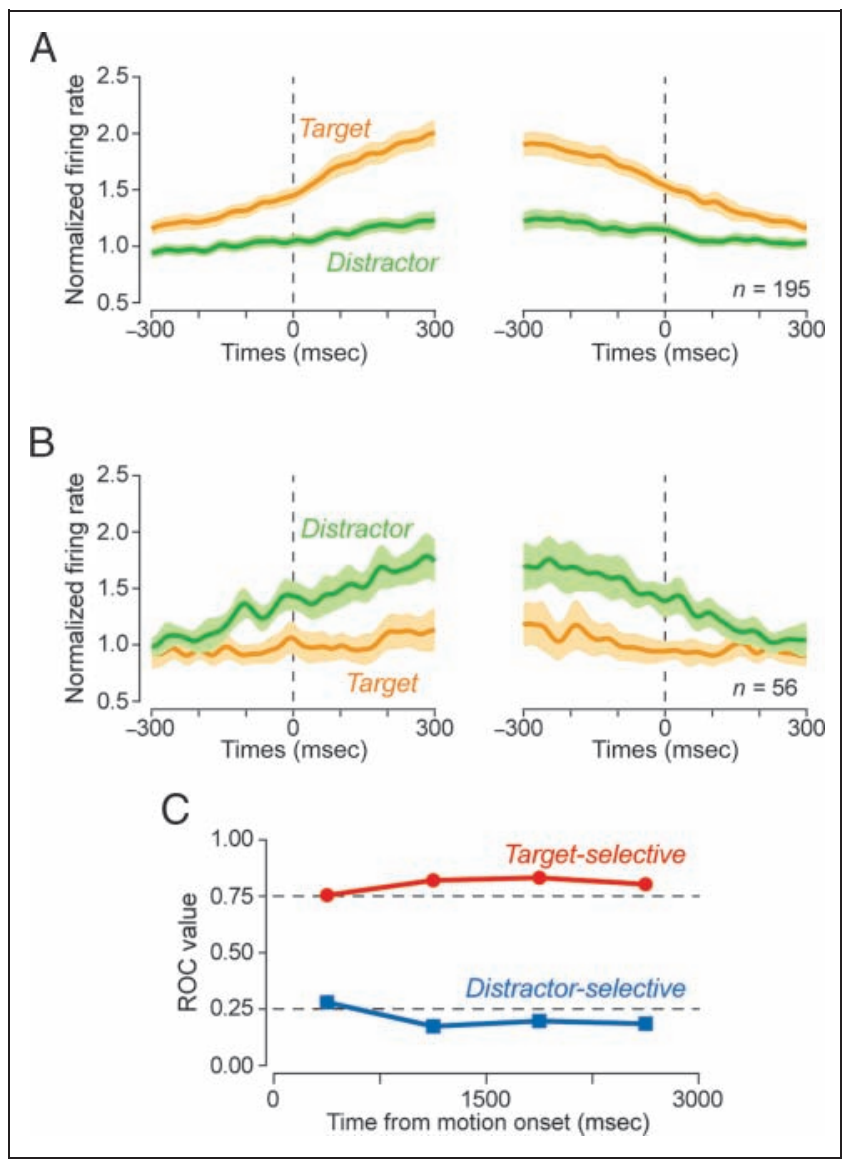

Figure 9. Dynamics of the object-selective response in the population of neurons. (A) Target-selective neurons. (B) Distractor-selective neurons. Time courses of population activity when the target (orange traces) or the distractor (green traces) moved toward (left) or away from (right) the $\mathrm{RF}$. For each neuron, the firing rates were normalized for the average activity during the entire motion period. Data are aligned with the time when each object crossed the border of the RF. Shaded areas indicate 95\% confidence intervals. (C) Neuronal discriminability of objects for different motion intervals. ROC values computed for each type of neurons did not vary throughout the motion period. The values of unity and zero indicate strong selectivity for the target and distractor, respectively. the average waveform) for individual neurons. The spike width did not differ between the target-selective type (358.5 $\pm 140.2 \mu \mathrm{sec}, n=39)$ and the distractor-selective type $(344.0 \pm 158.8 \mu \mathrm{sec}, n=25)$ neurons ( $t$ test, $p>.05)$.

\section{DISCUSSION}

To date, the suppression of irrelevant information has been thought to be a natural consequence of the enhancement of relevant information via mutual inhibition between conflicting representations (Reynolds \& Heeger, 2009; Desimone \& Duncan, 1995). This inherent property of neuronal networks might play an important role in accentuating the relevant information and explain various physiological data reported in studies of selective attention (Sundberg et al., 2009; Chen et al., 2008; McAdams \& Maunsell, 1999; Treue \& Maunsell, 1999; Hillyard et al., 1998; Luck et al., 1997; Motter, 1994; Schall \& Hanes, 1993; Moran \& Desimone, 1985). In addition to these passive and nonselective inhibitory processes, recent psychophysical experiments suggest that visual processing is also actively and selectively suppressed at the distractor location (Thomas \& Lleras, 2009; Flombaum et al., 2008; Pylyshyn et al., 2008; Pylyshyn, 2006; Ogawa et al., 2002; Muller \& von Muhlenen, 2000; Watson \& Humphreys, 2000; Klein, 1988). To address the underlying neuronal mechanisms, we examined the instantaneous firing rate of single neurons in the pre-FEF when monkeys covertly tracked a moving object in the presence of a visually identical, moving distractor.

Consistent with previous studies in V4 (Sundberg et al., 2009; Mitchell et al., 2007), we found that the majority of neurons in the pre-FEF responded more to the target than to the distractor. Given that each targetselective type neuron had its own RF, the target location at every moment can be represented precisely by the locus of activity in this neuronal population. Because electrical stimulation applied to the FEF is known to facilitate visual processing (Moore \& Fallah, 2004; Moore \& Armstrong, 2003), the activity selective to the target would plausibly enhance visual processing at the target location (Doran \& Hoffman, 2010; Drew, McCollough, Horowitz, \& Vogel, 2009). The present data are the first to demonstrate that signals highlighting the most relevant object are dynamically updated during covert object tracking.

In addition, we also found a novel type of neurons that exhibited greater firing modulation for the distractor than the target. Because each object trajectory was randomly controlled during experiments, the response to the distractor could not be accounted for by simple suppression from the target-selective type neurons. Importantly, the distractorselective activity disappeared for the green, explicit distractor that was effortlessly ignored by monkeys, whereas the same neurons responded to the original, implicit distractor that was indistinguishable from the target. These results demonstrate that the brain continuously monitors the distractor only when necessary to internally discriminate it 
from the target. Specific marking of the implicit distractor might be related to active suppression of visual processing reported previously (Thomas \& Lleras, 2009; Pylyshyn et al., 2008; Muller \& von Muhlenen, 2000; Klein, 1988) and could help to reduce the rate of erroneous choices during covert object tracking.

Several previous findings might be directly related to the hypothesis that the PFC is involved in the suppression of visual processing for distracting stimuli. A recent study showed that neurons in the pre-FEF exhibit transient activity during suppression of specific saccades (Hasegawa et al., 2004). Because the distractor-selective type neurons in this study exhibited discrete RF and because our recording sites anterior to the inferior limb of the arcuate sulcus (Figure 3) were similar to those reported in the previous study (Hasegawa et al., 2004), there might be some functional linkage between movement suppression and the specific marking of a moving distractor. Furthermore, other studies have shown that transcranial magnetic stimulation of the FEF results in topographically specific suppression of neuronal activity in early visual cortices (Ruff et al., 2006) and that ERPs evoked by the appearance of task-irrelevant stimuli increase in individuals with frontal lobe lesions (Chao \& Knight, 1995; Woods \& Knight, 1986). Because the distractor location was continuously represented by the population of distractor-selective type neurons, the signal might play a causal role in spatially specific suppression of visual processing at distractor locations during covert object tracking (Flombaum et al., 2008; Pylyshyn et al., 2008; Pylyshyn, 2006).

Recent studies have also isolated components of ERPs in humans related to the selective suppression of distractor. One component, known as the distractor positivity or Pd (Hickey, Di Lollo, \& McDonald, 2009), is recorded over the posterior contralateral sites and can be evoked by pop-out distractor (Kiss, Grubert, Petersen, \& Eimer, 2012; Sawaki \& Luck, 2010) or items sharing the target features (Sawaki \& Luck, 2011). Although neural sources of Pd have not been tested, its similar scalp distributions to N2pc suggest that it might originate from area $\mathrm{V} 4$ and the lateral occipital complex (Hopf et al., 2006; Hopf, Boelmans, Schoenfeld, Luck, \& Heinze, 2004). The other component, called frontal N2, is also evoked by distractors with strong preference for the objects similar to the target (Folstein \& Van Petten, 2008; Azizian, Freitas, Parvaz, \& Squires, 2006; Kenemans, Lijffijt, Camfferman, \& Verbaten, 2002). These previous studies and the present results are in line with the recent proposal that the frontal cortex is more involved in resolving internal conflict (Buschman \& Miller, 2007) whereas the posterior visual cortices represent features prioritized by the top-down signals (Itti \& Koch, 2001).

Why did neurons in the PFC respond only weakly to the green, explicit distractor? Although pop-out distractors have a potential to automatically capture attention (Theeuwes, 1991), the top-down factors could attenuate this distraction if the subjects are informed of target features and well trained to ignore the distractors that are distinct from the target (Folk \& Remington, 1998). Indeed, a previous study showed that the response of parietal neurons to a pop-out stimulus is greatly reduced when monkeys overtly ignore it (Ipata, Gee, Gottlieb, Bisley, \& Goldberg, 2006). Similarly, the response to the green distractor was greatly reduced in this study, likely because our monkeys adopt the strategy to ignore any green object. The top-down signals for the suppression of green objects might exist somewhere in the brain, but the signals were not detectable in this study that examined the spatial modulation of neuronal activity. Further studies are needed to elucidate neuronal signals for the nonspatial, feature-based selective suppression that must be necessary to ignore distractors in many daily situations.

In conclusion, this study revealed that neurons in the PFC provide multiple top-down signals for covert object tracking. One group of neurons represented the target location at every moment during object tracking, which could boost sensory processing at the target location (Moore \& Fallah, 2004; Moore \& Armstrong, 2003; Hopfinger, Buonocore, \& Mangun, 2000). The other group of neurons selectively responded to the distractor, suggesting an involvement in active suppression of visual processing at the distractor location (Thomas \& Lleras, 2009; Flombaum et al., 2008; Pylyshyn et al., 2008; Pylyshyn, 2006; Ogawa et al., 2002; Muller \& von Muhlenen, 2000; Watson \& Humphreys, 2000; Klein, 1988). Although the attentional modulation for the target might increase the hit rate of object choice, the signals related to the distractor might reduce the false alarm rate during covert tracking. Although we are usually unaware of the latter process, the efficient suppression of irrelevant information is necessary for high-level performance in various cognitive tasks (Lennert \& Martinez-Trujillo, 2011; Zanto \& Gazzaley, 2009; Vogel, McCollough, \& Machizawa, 2005; Kok, 1999; Smith \& Jonides, 1999; Dias, Robbins, \& Roberts, 1997), and the impairment of the process is thought to be responsible for the cognitive deficits observed in attention deficit/hyperactivity disorder (Friedman-Hill et al., 2010; Barkley, 1997), schizophrenia (Heinrichs \& Zakzanis, 1998), as well as elderly individuals (Gazzaley, Cooney, Rissman, \& D'Esposito, 2005; Hasher \& Zacks, 1988), all of which are associated with frontal lobe dysfunction.

\section{Acknowledgments}

We would like to thank Dr. Michael E. Goldberg for his comments on an early draft of this manuscript. We also appreciate many constructive comments from two anonymous reviewers. We are grateful to Tatsuya Shiraishi and Seiko Hirano for the animal care and training, Jun Kunimatsu for participating in the surgery, Mari Suzuki for administrative help, and all laboratory members for comments and discussions. This work was supported in part by grants from the Ministry of Education, Culture, Sports, Science, and Technology of Japan; the Ministry of Health, Labor and Welfare of Japan; and the Precursory Research for Embryonic Science and Technology, Japan Science and Technology Agency; the Takeda Foundation; the Uehara Memorial Foundation; and the Suhara Memorial Foundation. 
Reprint requests should be sent to Dr. Masaki Tanaka, Department of Physiology, Hokkaido University School of Medicine, North 15 , West 7, Sapporo 060-8638, Japan, or via e-mail: masaki@med. hokudai.ac.jp.

\section{REFERENCES}

Anderson, E. B., Mitchell, J. F., \& Reynolds, J. H. C. (2011). Attentional modulation of firing rate varies with burstiness across putative pyramidal neurons in macaque visual area V4. Journal of Neuroscience, 31, 10983-10992.

Azizian, A., Freitas, A. L., Parvaz, M. A., \& Squires, N. K. (2006). Beware misleading cues: Perceptual similarity modulates the N2/P3 complex. Psychophysiology, 43, 253-260.

Barkley, R. A. (1997). Behavioral inhibition, sustained attention, and executive functions: Constructing a unifying theory of ADHD. Psychological Bulletin, 121, 65-94.

Bruce, C. J., \& Goldberg, M. E. (1985). Primate frontal eye fields. I. Single neurons discharging before saccades. Journal of Neurophysiology, 53, 603-635.

Buschman, T. J., \& Miller, E. K. (2007). Top-down versus bottom-up control of attention in the prefrontal and posterior parietal cortices. Science, 315, 1860-1862.

Cepeda, N. J., Cave, K. R., Bichot, N. P., \& Kim, M. S. (1998). Spatial selection via feature-driven inhibition of distractor locations. Perception \& Psychophysics, 60, 727-746.

Chao, L. L., \& Knight, R. T. (1995). Human prefrontal lesions increase distractibility to irrelevant sensory inputs. NeuroReport, 6, 1605-1610.

Chen, Y., Martinez-Conde, S., Macknik, S. L., Bereshpolova, Y., Swadlow, H. A., \& Alonso, J. M. (2008). Task difficulty modulates the activity of specific neuronal populations in primary visual cortex. Nature Neuroscience, 11, 974-982.

Cohen, Y. E., \& Andersen, R. A. (2002). A common reference frame for movement plans in the posterior parietal cortex. Nature Reviews Neuroscience, 3, 553-562.

Corbetta, M., \& Shulman, G. L. (2002). Control of goal-directed and stimulus-driven attention in the brain. Nature Reviews Neuroscience, 3, 201-215.

Desimone, R. (1998). Visual attention mediated by biased competition in extrastriate visual cortex. Philosophical Transactions of the Royal Society of London, Series B, Biological Sciences, 353, 1245-1255.

Desimone, R., \& Duncan, J. (1995). Neural mechanisms of selective visual attention. Annual Review of Neuroscience, 18, 193-222.

Dias, R., Robbins, T. W., \& Roberts, A. C. (1997). Dissociable forms of inhibitory control within prefrontal cortex with an analog of the Wisconsin Card Sort Test: Restriction to novel situations and independence from "on-line" processing. Journal of Neuroscience, 17, 9285-9297.

Doran, M. M., \& Hoffman, J. E. (2010). The role of visual attention in multiple object tracking: Evidence from ERPs. Attention Perception \& Psychophysics, 72, 33-52.

Drew, T., McCollough, A. W., Horowitz, T. S., \& Vogel, E. K. (2009). Attentional enhancement during multiple-object tracking. Psychonomic Bulletin \& Review, 16, 411-417.

Flombaum, J. I., Scholl, B. J., \& Pylyshyn, Z. W. (2008). Attentional resources in visual tracking through occlusion: The high-beams effect. Cognition, 107, 904-931.

Folk, C. L., \& Remington, R. (1998). Selectivity in distraction by irrelevant featural singletons: Evidence for two forms of attentional capture. Journal of Experimental Psychology: Human Perception and Performance, 24, 847-858.

Folstein, J. R., \& Van Petten, C. (2008). Influence of cognitive control and mismatch on the N2 component of the ERP: A review. Psychophysiology, 45, 152-170.
Friedman-Hill, S. R., Wagman, M. R., Gex, S. E., Pine, D. S., Leibenluft, E., \& Ungerleider, L. G. C. (2010). What does distractibility in ADHD reveal about mechanisms for top-down attentional control? Cognition, 115, 93-103.

Gazzaley, A., Cooney, J. W., Rissman, J., \& D’Esposito, M. (2005). Top-down suppression deficit underlies working memory impairment in normal aging. Nature Neuroscience, 8, 1298-1300

Golomb, J. D., Chun, M. M., \& Mazer, J. A. (2008). The native coordinate system of spatial attention is retinotopic. Journal of Neuroscience, 28, 10654-10662.

Hasegawa, R. P., Peterson, B. W., \& Goldberg, M. E. (2004). Prefrontal neurons coding suppression of specific saccades Neuron, 43, 415-425.

Hasher, L., \& Zacks, R. T. (1988). Working memory, comprehension, and aging: A review and a new view. The Psychology of Learning and Motivation, 22, 193-225.

Heinrichs, R. W., \& Zakzanis, K. K. (1998). Neurocognitive deficit in schizophrenia: A quantitative review of the evidence. Neuropsychology, 12, 426-445.

Hickey, C., Di Lollo, V., \& McDonald, J. J. (2009). Electrophysiological indices of target and distractor processing in visual search. Journal of Cognitive Neuroscience, 21, 760-775.

Hillyard, S. A., Vogel, E. K., \& Luck, S. J. (1998). Sensory gain control (amplification) as a mechanism of selective attention: Electrophysiological and neuroimaging evidence. Philosophical Transactions of the Royal Society of London, Series B, Biological Sciences, 353, 1257-1270.

Hopf, J. M., Boehler, C. N., Luck, S. J., Tsotsos, J. K., Heinze, H. J., \& Schoenfeld, M. A. (2006). Direct neurophysiological evidence for spatial suppression surrounding the focus of attention in vision. Proceedings of the National Academy of Sciences, U.S.A., 103, 1053-1058.

Hopf, J. M., Boelmans, K., Schoenfeld, M. A., Luck, S. J., \& Heinze, H. J. (2004). Attention to features precedes attention to locations in visual search: Evidence from electromagnetic brain responses in humans. Journal of Neuroscience, 24, 1822-1832.

Hopfinger, J. B., Buonocore, M. H., \& Mangun, G. R. (2000). The neural mechanisms of top-down attentional control. Nature Neuroscience, 3, 284-291.

Ipata, A. E., Gee, A. L., Gottlieb, J., Bisley, J. W., \& Goldberg, M. E. (2006). LIP responses to a popout stimulus are reduced if it is overtly ignored. Nature Neuroscience, 9, 1071-1076.

Itti, L., \& Koch, C. (2001). Computational modelling of visual attention. Nature Reviews Neuroscience, 2, 194-203.

Kenemans, J. L., Lijffijt, M., Camfferman, G., \& Verbaten, M. N. (2002). Split-second sequential selective activation in human secondary visual cortex. Journal of Cognitive Neuroscience, 14, 48-61.

Kiss, M., Grubert, A., Petersen, A., \& Eimer, M. (2012). Attentional capture by salient distractors during visual search is determined by temporal task demands. Journal of Cognitive Neuroscience, 24, 749-759.

Klein, R. (1988). Inhibitory tagging system facilitates visual search. Nature, 334, 430-431.

Kok, A. (1999). Varieties of inhibition: Manifestations in cognition, event-related potentials and aging. Acta Psychologica (Amsterdam), 101, 129-158.

Lennert, T., \& Martinez-Trujillo, J. (2011). Strength of response suppression to distracter stimuli determines attentionalfiltering performance in primate prefrontal neurons. Neuron, 70, 141-152.

Luck, S. J., Chelazzi, L., Hillyard, S. A., \& Desimone, R. (1997). Neural mechanisms of spatial selective attention in areas V1, $\mathrm{V} 2$, and V4 of macaque visual cortex. Journal of Neurophysiology, 77, 24-42. 
Luck, S. J., Woodman, G. F., \& Vogel, E. K. (2000). Event-related potential studies of attention [Review]. Trends in Cognitive Sciences, 4, 432-440.

McAdams, C. J., \& Maunsell, J. H. (1999). Effects of attention on orientation-tuning functions of single neurons in macaque cortical area V4. Journal of Neuroscience, 19, 431-441.

Mitchell, J. F., Sundberg, K. A., \& Reynolds, J. H. (2007). Differential attention-dependent response modulation across cell classes in macaque visual area V4. Neuron, 55, 131-141.

Moore, T., \& Armstrong, K. M. (2003). Selective gating of visual signals by microstimulation of frontal cortex. Nature, 421, 370-373

Moore, T., \& Fallah, M. (2004). Microstimulation of the frontal eye field and its effects on covert spatial attention. Journal of Neurophysiology, 91, 152-162.

Moran, J., \& Desimone, R. (1985). Selective attention gates visual processing in the extrastriate cortex. Science, 229, 782-784.

Motter, B. C. (1994). Neural correlates of attentive selection for color or luminance in extrastriate area V4. Journal of Neuroscience, 14, 2178-2189.

Muller, H. J., \& von Muhlenen, A. (2000). Probing distractor inhibition in visual search: Inhibition of return. Journal of Experimental Psychology: Human Perception and Performance, 26, 1591-1605.

Ogawa, H., Takeda, Y., \& Yagi, A. (2002). Inhibitory tagging on randomly moving objects. Psychological Science, 13, 125-129.

Polk, T. A., Drake, R. M., Jonides, J. J., Smith, M. R., \& Smith, E. E. C. (2008). Attention enhances the neural processing of relevant features and suppresses the processing of irrelevant features in humans: A functional magnetic resonance imaging study of the Stroop task. Journal of Neuroscience, 28, 13786-13792.

Posner, M. I., \& Petersen, S. E. (1990). The attention system of the human brain. Annual Review of Neuroscience, 13, 25-42.

Pouget, P., Emeric, E. E., Stuphorn, V., Reis, K., \& Schall, J. D. (2005). Chronometry of visual responses in frontal eye field, supplementary eye field, and anterior cingulate cortex. Journal of Neurophysiology, 94, 2086-2092.

Pylyshyn, Z. W. (2006). Some puzzling findings in multiple object tracking (MOT): II. Inhibition of moving nontargets. Visual Cognition, 14, 175-198.

Pylyshyn, Z. W., Haladjian, H. H., King, C. E., \& Reilly, J. E. (2008). Selective nontarget inhibition in multiple object tracking. Visual Cognition, 16, 1011-1021.

Pylyshyn, Z. W., \& Storm, R. W. (1988). Tracking multiple independent targets: Evidence for a parallel tracking mechanism. Spatial Vision, 3, 179-197.
Reynolds, J. H., \& Heeger, D. J. (2009). The normalization model of attention. Neuron, 61, 168-185.

Ruff, C. C., Blankenburg, F., Bjoertomt, O., Bestmann, S., Freeman, E., Haynes, J. D., et al. (2006). Concurrent TMS-fMRI and psychophysics reveal frontal influences on human retinotopic visual cortex. Current Biology, 16, 1479-1488.

Sawaki, R., \& Luck, S. J. (2010). Capture versus suppression of attention by salient singletons: Electrophysiological evidence for an automatic attend-to-me signal. In Attention, Perception, \& Psychophysics, 72, 1455-1470.

Sawaki, R., \& Luck, S. J. (2011). Active suppression of distractors that match the contents of visual working memory. Visual Cognition, 19, 956-972.

Schall, J. D., \& Hanes, D. P. (1993). Neural basis of saccade target selection in frontal eye field during visual search. Nature, 366, 467-469.

Smith, E. E., \& Jonides, J. (1999). Storage and executive processes in the frontal lobes. Science, 283, 1657-1661.

Sundberg, K. A., Mitchell, J. F., \& Reynolds, J. H. (2009). Spatial attention modulates center-surround interactions in macaque visual area v4. Neuron, 61, 952-963.

Theeuwes, J. (1991). Cross-dimensional perceptual selectivity. Perception \& Psychophysics, 50, 184-193.

Thomas, L. E., \& Lleras, A. (2009). Inhibitory tagging in an interrupted visual search. Attention, Perception, \& Psychophysics, 71, 1241-1250.

Thompson, K. G., Hanes, D. P., Bichot, N. P., \& Schall, J. D. (1996). Perceptual and motor processing stages identified in the activity of macaque frontal eye field neurons during visual search. Journal of Neurophysiology, 76, 4040-4055.

Treue, S., \& Maunsell, J. H. (1999). Effects of attention on the processing of motion in macaque middle temporal and medial superior temporal visual cortical areas. Journal of Neuroscience, 19, 7591-7602.

Vogel, E. K., McCollough, A. W., \& Machizawa, M. G. (2005). Neural measures reveal individual differences in controlling access to working memory. Nature, 438, 500-503.

Watson, D. G., \& Humphreys, G. W. (2000). Visual marking: Evidence for inhibition using a probe-dot detection paradigm. Perception \& Psychophysics, 62, 471-481.

Woods, D. L., \& Knight, R. T. (1986). Electrophysiologic evidence of increased distractibility after dorsolateral prefrontal lesions. Neurology, 36, 212-216.

Zanto, T. P., \& Gazzaley, A. (2009). Neural suppression of irrelevant information underlies optimal working memory performance. Journal of Neuroscience, 29, 3059-3066. 